\title{
Tendencias de mortalidad por traumatismos y envenenamientos en adolescentes. México, 1979-1997
}

\author{
Alfredo C elis, MC, MSP, Dr en Epidemiología, (1,2) Zoila Gómez-Lomelí, MC, M en Gerontología Social, (2) \\ Jesús Armas, Lic en Econ. (3)
}

\section{Celis A, Gómez-Lomelí Z,Armas J. Tendencias de mortalidad por traumatismos y envenenamientos en adolescentes. México, 1979-1997. Salud Publica Mex 2003;45 supl 1:S8-S15.}

\section{Resumen}

Objetivo. Describir las causas externas de la mortalidad por traumatismos y envenenamientos, así como la tendencia que estas causas han mostrado entre adolescentes en México, de 1979 a 1997. Material y métodos. Para la realización de este estudio descriptivo de la mortalidad se utilizaron las bases de datos de mortalidad registrada en México de 1979 a 1997. Los datos son recabados, codificados y capturados por el Instituto N acional de Estadística, Geografía e Informática, y se obtuvieron a través del Instituto $\mathrm{N}$ acional de Salud Pública. Los datos fueron tabulados durante el último semestre de 2000 , en Jalisco, en la U nidad de Investigación Epidemiológica y en Ser vicios de Salud del Adolescente del Instituto Mexicano del Seguro Social. Resultados. Los traumatismos y envenenamientos ocuparon el primer lugar en la mortalidad del grupo adolescente (tasa de 13.35/100 000) con una tendencia descendente de -41.4\% durante el periodo 1979-1997, no obstante el incremento de homicidios y suicidios $(9.5 \%$ y $104.0 \%$ respectivamente). Los mecanismos más frecuentes fueron los vehículos de tráfico de motor, las armas de fuego, las intoxicaciones, las sofocaciones y las asfixias por inmersión. Conclusiones. Los traumatismos y envenenamientos que experimenta la población de países en desarrollo muestran frecuencias diferentes a lo registrado en los más desarrollados, haciendo necesario el estudio de los mismos para implantar las medidas preventivas acordes con las condiciones socioculturales y ambientales de cada entorno.

Palabras clave: mortalidad/tendencias; traumatismos; envenenamiento; adolescencia; accidentes; violencia; M éxico

\author{
CelisA, Gómez-Lomelí Z,Armas J. \\ Adolescent injury \\ and poisoning mortality trends. \\ Mexico, 1979-1997. \\ Salud Publica Mex 2003;45 suppl 1:S8-S15.
}

\begin{abstract}
A bstract
Objective.To describe the external causes of death and mortality trends due to injuries and poisoning in Mexican adolescents, from 1979 to 1997. Material and Methods. This is a descriptive study of deaths occurring in Mexico from 1979 to 1997. D ata were abstracted, coded, and entered in electronic format, by Instituto $N$ acional de Estadística, G eografía e Informática (N ational Institute of Statistics, Geography, and Informatics, IN EGI). Data were analyzed during the second half of year 2000, in Jalisco, at the Research U nit of Epidemiologic and Adolescent Health Services, of Instituto Mexicano del Seguro Social (Mexican Institute of So cial Security, IMSS). Results. Injuries and poisoning ranked first as causes of adolescent mortality in Mexico (rate $=13.35 / 100,000$ ), decreasing $41.4 \%$ from 1979 to 1997. However, an increasing trend was observed for homicides and suicides ( $9.5 \%$ y $104.0 \%$, respectively). The most frequent mechanisms of injury were: motor vehicles, firearms, intoxications, suffocation, and drowning. Conclusions. Injuries and poisoning in developing countries show differences from those reported in developed countries. Further studies should be conducted to find preventive measures consistent with the sociocultural and environmental determinants of injuries and poisoning.
\end{abstract}

Key words: mortality/trends; injury; poisoning; adolescence; accidents; violence; mortality; México

(1) Unidad de Investigación Epidemiológica y en Servicios de Salud del Adolescente, Instituto Mexicano del Seguro Social. Guadalajara, Jalisco, México.

(2) Departamento de Salud Pública, Centro Universitario de Ciencias de la Salud, Universidad de Guadalajara. Guadalajara, Jalisco, México.

(3) Dirección de Estadística, Instituto N acional de Estadística, Geografía e Informática. Guadalajara, Jalisco, México.

Fecha de recibido: 18 de abril de 2001 - Fecha de aprobado: 28 de agosto de 2002 Solicitud de sobretiros: Alfredo Celis. Avenida N aciones Unidas 6754-9B,Virreyes Residencial, 45110, Zapopan, Jalisco, México. Correo electrónico: alfredo_celis@yahoo.com 
os traumatismos y los envenenamientos (TyE) se encuentran en el primer lugar de los listados de causas de muerte en los adolescentes, ${ }^{1}$ y poco se han estudiado en este grupo de edad. Su estudio es importante porque la adolescencia es una etapa de la vida en la que el sujeto experimenta una gran cantidad de cambios biopsicosociales que lo diferencian de los grupos etarios previo y posterior. No obstante, las estadísticas nacionales de mortalidad no atienden estas diferencias y nos presentan cifras con agrupaciones de 5 a 14 y de 15 a 24 años de edad, ${ }^{2}$ las cuales no permiten particularizar las características de la mortalidad observada durante la adolescencia temprana (10 a 14 años) y tardía (15 a 19 años). Por este motivo nuestro trabajo busca describir la mortalidad por TyE, y su tendencia, que los adolescentes en México han experimentado de 1979 a 1997.

\section{Material y métodos}

Para este trabajo se utilizaron las bases de datos de mortalidad registrada en México de 1979 a 1997. Los datos son recabados, codificados y capturados por el Instituto Nacional de Geografía, Estadística e Informática, y se obtuvieron a través del Instituto Nacional de Salud Pública. Del total de registros sólo se tabularon los correspondientes al grupo de 10 a 19 años de edad. Al momento de seleccionar los registros se puso especial cuidado en la codificación original de la edad, pues durante el periodo estudiado esta variable fue codificada de tres maneras diferentes: edad en años, edad especificando años mediante la letra " $\mathrm{A}$ ", y edad especificando años mediante el carácter " 4 ". Una vez conformada la base de datos de defunciones en adolescentes, el análisis se restringió a las siguientes variables: sexo (masculino, femenino), grupo de edad (10 a 14 y 15 a 19 años), causa de muerte según las categorías de tres y cuatro dígitos del anexo E de la clasificación internacional de enfermedades, 9a. revisión, y año de registro. Las defunciones se agruparon según los criterios del Centro de Prevención y Control de Enfermedades (CDC, por sus siglas en inglés) expresados en la matriz que se presenta en la dirección de internet http://www.cdc.gov/ncipc/whatsnew/ matrix $2 . h t m$

El análisis estadístico incluyó el cálculo de tasas de mortalidad (número de defunciones en el periodo dividido entre el total de años persona acumulados por la población en el mismo periodo $)^{3}$ para cada año estudiado. En grupos con crecimiento estable la población a mitad de cada año es igual al total de años persona durante ese periodo: este número se utilizó como denominador para el cálculo de las tasas de mor- talidad. El denominador de las tasas se proyectó linealmente $^{4}$ a partir de la población adolescente registrada en los Censos Generales de Población de 1980 y 1990, y en el Conteo General de Población de 1995. Las tendencias de mortalidad se estimaron mediante regresión simple. Se consideró que una tendencia era estadísticamente significativa si el valor de $p$ correspondiente a la pendiente era menor o igual a 0.05. Para simplificar su presentación tabular y ahorrar espacio en la publicación del trabajo, la mortalidad observada se agrupó en tres periodos: 1979-1981, 1987-1989 y 19951997. Esta agrupación favorece la estabilidad de las tasas de mortalidad, particularmente cuando los números absolutos son pequeños. Para estimar la mortalidad de cada trienio se sumaron las tasas correspondientes a los tres años y se dividieron entre tres. ${ }^{4}$ Los cambios porcentuales se estimaron a partir de estas agrupaciones. La tabulación se realizó según grupo de edad y sexo: la correspondiente a cada sexo se presenta textualmente en la sección de resultados; la que distingue grupo de edad y sexo se presenta en los cuadros I y II. Debido a las restricciones que limitan el espacio para este tipo de publicaciones, los cuadros correspondientes a los resultados para cada año no se presentan en este documento, pero están a disposición de quien lo solicite a los autores.

\section{Resultados}

En México, de 1979 a 1997 se registraron 152570 defunciones por TyE en adolescentes. Durante el periodo 79-97 la mortalidad por TyE mostró una tendencia descendente significativa $(p<0.01)$ en todos los grupos (figura 1), siendo la reducción global de $-41.5 \%$ (de 55.9/100 000 en el trienio 79-81, a 32.7/100 000 en el trienio 95-97). Esta tendencia es más marcada en el grupo de 10 a 14 años (-52.13\% en hombres, $-47.01 \%$ en mujeres) que en el grupo de 15 a 19 años $(-40.79 \%$ en hombres, $-33.97 \%$ en mujeres). En los cuatro grupos los TyE ocuparon el primer lugar entre las causas de muertes (figura 2), representando durante todo el periodo $54.5 \%$ de todas las muertes, y manteniendo durante los 19 años estudiados una proporción en relación con el total de muertes más o menos constante al interior de cada grupo; así, los TyE representaron, aproximadamente, $30 \%$ de las muertes en mujeres de 10 a 14 años, 31\% en las mujeres de 15 a 19 años, 52\% en los hombres de 10 a 14, y 71\% en los hombres de 15 a 19 años. Al final del periodo, durante el trienio 1995-1997, la tasa de mortalidad entre los adolescentes mostró diferencias importantes según grupo de edad y sexo: en mujeres de 10 a 14 años fue de 9.21/100 000; en mujeres de 15 a 19 años fue de 17.59/100 000; en hombres 


\section{Cuadro I}

\section{Tasas de mortalidad por traumatismos y envenenamientos en mujeres adolescentes en México.} TrIENIOS 1979-1981, 1987-1989 Y 1995-1997

\begin{tabular}{|c|c|c|c|c|c|c|c|}
\hline & & & 0 a 14 año & & & a 19 años & \\
\hline Causa & Código & $79-81$ & $87-89$ & $95-97$ & $79-81$ & $87-89$ & $95-97$ \\
\hline Todas las causas de muerte & 001-999 & 56.19 & 39.92 & 29.50 & 87.10 & 57.80 & 49.00 \\
\hline $\begin{array}{l}\text { Todas las causas externas* } \\
\text { Tráfico, vehículo de motor (TVM) }\end{array}$ & E800-E999 & 17.38 & 11.97 & 9.21 & 26.67 & 17.65 & 17.59 \\
\hline No intencional & E810-E819 & 5.52 & 3.70 & 3.30 & 7.78 & 4.67 & 5.39 \\
\hline Persona & (4º digito) & & & & & & \\
\hline 0 cupante & $0, .1$ & 0.07 & 0.03 & 0.16 & 0.17 & 0.06 & 0.14 \\
\hline Motociclista & $.2, .3$ & 0.00 & 0.01 & 0.01 & 0.05 & 0.00 & 0.06 \\
\hline Ciclista & .6 & 0.00 & 0.01 & 0.02 & 0.00 & 0.00 & 0.01 \\
\hline Peatón & .7 & 1.33 & 1.40 & 1.47 & 1.34 & 1.42 & 1.85 \\
\hline No especificado & .9 & 4.12 & 2.26 & 1.64 & 6.21 & 3.18 & 3.31 \\
\hline TVM, total & +E958.5, E988.5 & 5.52 & 3.70 & 3.30 & 7.78 & 4.67 & 5.39 \\
\hline Peatón, otro & E800-E807 (.2), & 0.00 & 0.00 & 0.03 & 0.00 & 0.05 & 0.03 \\
\hline & $\begin{array}{l}\text { E820-E825 (.7), } \\
\text { E826-E829 (.0) }\end{array}$ & & & & & & \\
\hline Arma de fuego, total & & 0.75 & 0.99 & 0.64 & 2.51 & 2.44 & 2.68 \\
\hline No intencional & E922 & 0.27 & 0.30 & 0.17 & 0.64 & 0.49 & 0.34 \\
\hline Suicidio & E955(.0-.4) & 0.01 & 0.08 & 0.07 & 0.27 & 0.38 & 0.66 \\
\hline Homicidio & E965(.0-.4), E970 & 0.35 & 0.57 & 0.32 & 1.30 & 1.49 & 1.48 \\
\hline Se ignora intención & E985(.0-.4) & 0.12 & 0.03 & 0.08 & 0.29 & 0.09 & 0.20 \\
\hline Inmersión, total & & 2.89 & 1.56 & 1.20 & 2.73 & 1.58 & 1.20 \\
\hline No intencional & E830, E832, E910 & 2.31 & 1.44 & 1.06 & 2.07 & 1.46 & 0.96 \\
\hline Suicidio & E954 & 0.01 & 0.00 & 0.00 & 0.03 & 0.00 & 0.03 \\
\hline Homicidio & E964 & 0.05 & 0.05 & 0.07 & 0.14 & 0.06 & 0.12 \\
\hline Se ignora intención & E984 & 0.52 & 0.07 & 0.07 & 0.48 & 0.06 & 0.08 \\
\hline Q uemaduras & & & & & & & \\
\hline No intencional & E890-E899 & 0.36 & 0.28 & 0.15 & 0.53 & 0.38 & 0.17 \\
\hline Total & +E858.1, E868.0, & 0.55 & 0.30 & 0.21 & 0.76 & 0.50 & 0.22 \\
\hline & E988.1 & & & & & & \\
\hline Sofocación, total & & 0.44 & 0.44 & 0.71 & 0.87 & 0.95 & 1.70 \\
\hline No intencional & & & & & & & \\
\hline Inhalación/ingestión & E911-E912 & 0.10 & 0.19 & 0.16 & 0.15 & 0.27 & 0.15 \\
\hline 0 tra & E913 & 0.18 & 0.10 & 0.11 & 0.23 & 0.16 & 0.19 \\
\hline Suicidio & E953 & 0.04 & 0.03 & 0.20 & 0.21 & 0.26 & 0.75 \\
\hline Homicidio & E963 & 0.05 & 0.10 & 0.20 & 0.10 & 0.22 & 0.51 \\
\hline Se ignora intención & E983 & 0.07 & 0.02 & 0.03 & 0.16 & 0.05 & 0.10 \\
\hline Caídas & & & & & & & \\
\hline No intencional & E880-E886, E888 & 0.19 & 0.25 & 0.22 & 0.15 & 0.21 & 0.19 \\
\hline Total & +E957, E968.1, E987 & 0.20 & 0.25 & 0.23 & 0.20 & 0.21 & 0.25 \\
\hline Cortante/punzante & & & & & & & \\
\hline Homicidio & E966, E974 & 0.15 & 0.25 & 0.20 & 0.47 & 0.40 & 0.57 \\
\hline Total & +E920, E956, E986 & 0.18 & 0.28 & 0.22 & 0.60 & 0.43 & 0.63 \\
\hline Intoxicación, total & & 0.62 & 0.53 & 0.68 & 2.24 & 1.52 & 2.07 \\
\hline No intencional & E850-E869 & 0.41 & 0.35 & 0.29 & 0.89 & 0.74 & 0.72 \\
\hline Suicidio & E950-E952 & 0.04 & 0.11 & 0.25 & 0.85 & 0.55 & 1.03 \\
\hline Homicidio & E962, E972 & 0.04 & 0.01 & 0.05 & 0.09 & 0.06 & 0.08 \\
\hline Se ignora intención & E980-E982 & 0.13 & 0.05 & 0.09 & 0.40 & 0.18 & 0.25 \\
\hline Totales & & & & & & & \\
\hline No intencional & E800-E949 & 13.74 & 9.95 & 7.07 & 18.66 & 12.60 & 10.54 \\
\hline Suicidio & E950-E959 & 0.13 & 0.27 & 0.53 & 1.59 & 1.30 & 2.55 \\
\hline Homicidio & E960-E978 & 1.05 & 1.41 & 1.16 & 2.68 & 3.10 & 3.40 \\
\hline Se ignora intención/otro & E980-E999 & 2.46 & 0.34 & 0.45 & 3.73 & 0.65 & 1.10 \\
\hline
\end{tabular}

Las frecuencias absolutas son: Grupo 10 a 14 años: 79-81, 2 356; 87-89, 1 807; 95-97, 1 461. Grupo 15 a 19 años: 79-81, 3 112; 87-89, 2 490; 95-97, 2 725 


\section{Cuadro II}

\section{TASAS de MORTALIDAd POR tRAumatismos y enVenenAmientos en hombres adolescentes en México. TRIENIOS 1979-1981, 1987-1989 Y 1995-1997}

\begin{tabular}{|c|c|c|c|c|c|c|c|}
\hline & & & 0 a 14 a & & & 5 a 19 añ & \\
\hline Causa & Código & $79-81$ & $87-89$ & $95-97$ & $79-81$ & $87-89$ & $95-97$ \\
\hline Todas las causas de muerte & 001-999 & 89.12 & 65.52 & 44.66 & 198.75 & 147.53 & 117.91 \\
\hline $\begin{array}{l}\text { Todas las causas externas* } \\
\text { Tráfico, vehículo de motor (TVM) }\end{array}$ & E800-E999 & 46.90 & 34.51 & 22.45 & 141.18 & 104.64 & 83.59 \\
\hline No intencional & E810-E819 & 13.25 & 9.43 & 7.02 & 33.07 & 21.46 & 21.44 \\
\hline Persona & (4º digito) & & & & & & \\
\hline 0 cupante & 0,1 & 0.17 & 0.05 & 0.26 & 1.18 & 0.23 & 0.45 \\
\hline Motociclista & $.2,3$ & 0.07 & 0.03 & 0.06 & 0.44 & 0.32 & 0.48 \\
\hline Ciclista & .6 & 0.07 & 0.05 & 0.10 & 0.11 & 0.07 & 0.28 \\
\hline Peatón & .7 & 3.65 & 4.37 & 3.85 & 5.51 & 5.89 & 7.13 \\
\hline No especificado & .9 & 9.26 & 4.94 & 2.74 & 25.82 & 14.92 & 13.07 \\
\hline TVM, total & +E958.5, E988.5 & 13.25 & 9.43 & 7.02 & 33.07 & 21.46 & 21.44 \\
\hline Peatón, otro & E800-E807 (.2), & 0.00 & 0.08 & 0.06 & 0.01 & 0.27 & 0.28 \\
\hline & E820-E825 (.7), & & & & & & \\
\hline & E826-E829 (.0) & & & & & & \\
\hline Arma de fuego, total & & 3.93 & 3.64 & 2.35 & 24.13 & 24.87 & 20.66 \\
\hline No intencional & E922 & 1.42 & 1.19 & 0.56 & 6.01 & 4.41 & 2.12 \\
\hline Suicidio & $\mathrm{E} 955(.0-.4)$ & 0.06 & 0.18 & 0.15 & 0.94 & 1.47 & 1.85 \\
\hline Homicidio & E965(.0-.4), E970 & 1.90 & 2.14 & 1.47 & 14.02 & 18.04 & 15.58 \\
\hline Se ignora intención & $E 985(.0-.4)$ & 0.55 & 0.13 & 0.17 & 3.17 & 0.95 & 1.11 \\
\hline Inmersión, total & & 8.38 & 5.80 & 3.91 & 18.99 & 13.34 & 8.67 \\
\hline No intencional & E830, E832, E910 & 6.62 & 5.36 & 3.47 & 14.81 & 12.23 & 7.29 \\
\hline Suicidio & E954 & 0.01 & 0.00 & 0.01 & 0.05 & 0.05 & 0.05 \\
\hline Homicidio & E964 & 0.18 & 0.25 & 0.20 & 0.66 & 0.50 & 0.59 \\
\hline Se ignora intención & E984 & 1.57 & 0.19 & 0.23 & 3.46 & 0.56 & 0.74 \\
\hline Q uemaduras & & & & & & & \\
\hline No intencional & E890-E899 & 0.74 & 0.52 & 0.23 & 1.45 & 0.89 & 0.54 \\
\hline Total & +E858.1, E868.0, & 0.96 & 0.56 & 0.26 & 2.03 & 0.95 & 0.63 \\
\hline & E988.1 & & & & & & \\
\hline Sofocación, total & & 1.37 & 1.50 & 2.07 & 4.34 & 4.08 & 6.34 \\
\hline No intencional & & & & & & & \\
\hline Inhalación/ingestión & E911-E912 & 0.20 & 0.33 & 0.27 & 0.35 & 0.61 & 0.45 \\
\hline 0 tra & E913 & 0.56 & 0.46 & 0.40 & 1.42 & 1.01 & 0.64 \\
\hline Suicidio & E953 & 0.31 & 0.45 & 0.92 & 1.44 & 1.60 & 3.75 \\
\hline Homicidio & E963 & 0.13 & 0.17 & 0.31 & 0.40 & 0.62 & 1.08 \\
\hline Se ignora intención & E983 & 0.17 & 0.09 & 0.17 & 0.72 & 0.24 & 0.41 \\
\hline Caídas & & & & & & & \\
\hline No intencional & E880-E886, E888 & 0.68 & 0.82 & 0.58 & 1.73 & 1.56 & 1.31 \\
\hline Total & +E957, E968.1, E987 & 0.69 & 0.83 & 0.61 & 1.82 & 1.60 & 1.44 \\
\hline Cortante/punzante & & & & & & & \\
\hline Homicidio & E966, E974 & 0.41 & 0.61 & 0.45 & 5.86 & 6.18 & 5.42 \\
\hline Total & +E920, E956, E986 & 0.56 & 0.75 & 0.53 & 8.04 & 7.37 & 5.96 \\
\hline Intoxicación, total & & 0.55 & 0.59 & 0.38 & 2.29 & 1.62 & 1.73 \\
\hline No intencional & E850-E869 & 0.36 & 0.48 & 0.26 & 1.39 & 1.03 & 1.00 \\
\hline Suicidio & E950-E952 & 0.03 & 0.05 & 0.06 & 0.42 & 0.35 & 0.30 \\
\hline Homicidio & E962, E972 & 0.04 & 0.01 & 0.03 & 0.06 & 0.07 & 0.11 \\
\hline Se ignora intención & E980-E982 & 0.12 & 0.06 & 0.04 & 0.42 & 0.17 & 0.32 \\
\hline Totales & & & & & & & \\
\hline No intencional & E800-E949 & 36.91 & 29.04 & 17.36 & 93.40 & 67.82 & 47.35 \\
\hline Suicidio & E950-E959 & 0.47 & 0.71 & 1.14 & 3.18 & 3.76 & 6.14 \\
\hline Homicidio & E960-E978 & 3.40 & 4.00 & 2.99 & 24.80 & 29.86 & 25.96 \\
\hline Se ignora intención/otro & E980-E999 & 6.13 & 0.75 & 0.96 & 19.81 & 3.20 & 4.14 \\
\hline
\end{tabular}

Las frecuencias absolutas son: Grupo 10 a 14 años:79-81, 6 437; 87-89, 5 107;95-97,3 663. G rupo 15 a 19 años:79-81, 15 953; 87-89, 14 273;95-97, 12 726 


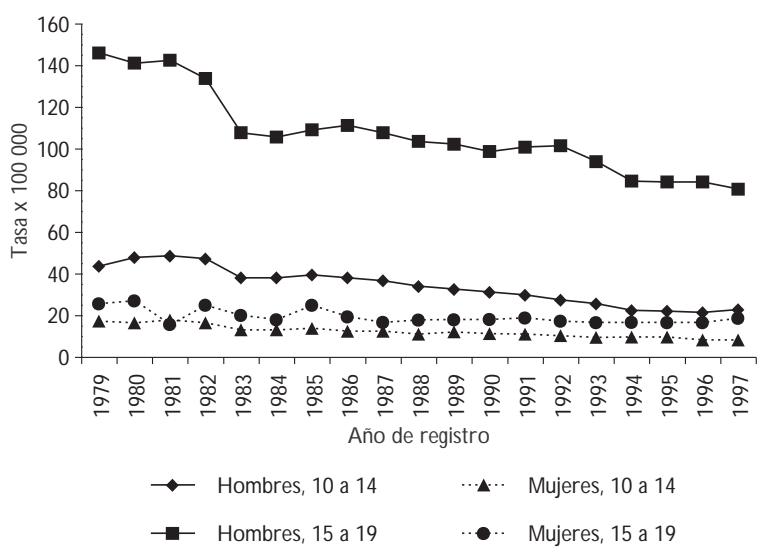

Figura 1.Tendencias de MORTALIDAD POR TRAUMATISMOS $Y$ ENVENENAMIENTOS EN ADOLESCENTES, SEGÚN GRUPO DE EDAD Y SEXO. MÉXICO, 1979-1997

de 10 a 14 años fue de 22.45/100 000, y en hombres de 15 a 19 años fue de 83.59/100 000 .

\section{Traumatismos y envenenamientos según su mecanismo}

Traumatismos producidos durante el tráfico de vehículo de motor

Estos eventos se presentan como la primera causa de muerte en todos los grupos. La tendencia general de los accidentes de tráfico de vehículo de motor (ATVM) ha sido descendente $(p<0.01)$, con una disminución general de $-36.9 \%$ entre adolescentes; la reducción observada del trienio 79-81 al 87-89 ha sido más importante, con una reducción de $33.5 \%$, que la apreciada del 87-89 al 95-97, donde la reducción fue apenas de $5.1 \%$. Esta tendencia descendente ha sido más consistente para los grupos de 10 a 14 años que para los de 15 a 19 años, donde se observan muchas variaciones en las tasas de mortalidad.

La gran mayoría de las muertes en ATVM correspondieron a peatones: la razón peatón/ocupante es de 9.2 para mujeres de 10 a 14 años de edad, 13.2 para mujeres de 15 a 19 años de edad, 14.8 para hombres de 10 a 14 años de edad, y de 15.8 para hombres de 15 a 19 años de edad. El registro de estos eventos muestra una tendencia ascendente, habiendo un incremento de 20\% durante el periodo estudiado. Estos son seguidos, en una cantidad mucho menor, por el registro de defunciones ocurridas en ATVM a pasajeros de vehículos (no motocicleta) y conductores, los cuales también

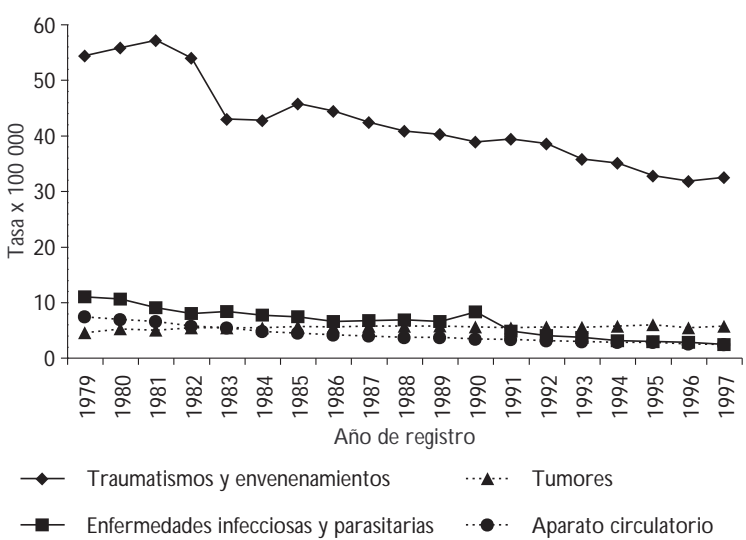

Figura 2.Tendencias de MORTALIDAD de LAS PRIMERAS CUATRO CAUSAS MÁs FRECUENTES EN ADOLESCENTES. MÉxıco, 1979-1997

muestran una tendencia ascendente. Es de notar que la categoría "no especificado" correspondiente a los ATVM muestra una tendencia descendente de $53.7 \%$.

\section{Traumatismos con armas de fuego}

Las defunciones producidas por proyectiles de arma de fuego mostraron una tendencia ascendente durante el periodo, de $79-81$ a $87-89$, de $4.5 \%$, y un descenso de $87-89$ a $95-97$ de $-16.3 \%$. Estas tendencias difieren según la intencionalidad del evento. Para los traumatismos no intencionales se observa una disminución de aproximadamente $-23.3 \%$ entre adolescentes. Por otra parte, para los suicidios y los homicidios se observan incrementos de $176.9 \%$ y $13.9 \%$, respectivamente.

\section{Intoxicaciones}

De manera general, las defunciones por intoxicación disminuyeron entre 79-89 y 87-89 para aumentar entre $87-89$ y 95-97. Los eventos no intencionales mostraron una ligera tendencia descendente $(p>0.05)$, que durante el periodo estudiado fue de $-23.3 \%$. Los tóxicos que con mayor frecuencia se observaron fueron "otras sustancias sólidas y líquidas" (representando de 41 a $56 \%$ de las fatalidades), seguidos por "gases y vapores" (de 30 a $42 \%$ ) y "drogas, medicamentos y productos biológicos" (de 9 a 17\%). Por otra parte, las intoxicaciones suicidas mostraron una disminución entre $79-81$ a $87-89$ de $-16.1 \%$, y un incremento entre $87-89$ a $95-97$ de $57.7 \%$. 


\section{Sofocaciones}

Las muertes a causa de sofocación mostraron una tendencia ascendente, pues su incremento fue de $59.2 \%$. Esta tendencia fue motivada por el incremento del ahorcamiento como medio de suicidio (que explica $51.7 \%$ del total de las muertes por este mecanismo) y homicidios, donde el incremento fue de $193.6 \%$, y $205.9 \%$, respectivamente. Las sofocaciones accidentales por inhalación de cuerpo extraño o alimento muestran una tendencia estable, aunque se observa que de un año a otro existen variaciones importantes en su frecuencia. Por otra parte, las sofocaciones por otras causas presentan una tendencia descendente $(p<0.01)$, con un cambio porcentual de $-42.9 \%$ del trienio 1997 1981 al 1997-1997.

\section{Inmersión accidental}

La asfixia por inmersión representa la segunda causa de muerte accidental en todos los grupos estudiados, y sólo es superada por los ATVM. Su tendencia general es descendente $(p<0.01)$, con una reducción de $53.7 \%$.

\section{Quemaduras}

Las quemaduras mortales muestran una tendencia descendente $(p<0.01)$ de $64.1 \%$ en el periodo estudiado, siendo más o menos semejante en los cuatro grupos etarios.

\section{Caídas}

Las caídas accidentales muestran una tendencia estable, aunque se observa que de un año a otro existen variaciones importantes en su frecuencia.

\section{Traumatismos y envenenamientos según su intención}

\section{Eventos no intencionales}

Los TyE mortales que resultaron de eventos no intencionales, o accidentales, han mostrado una tendencia descendente significativa $(p<0.01)$ de $-48.4 \%$ durante los 19 años estudiados. Esta tendencia es ligeramente más marcada entre los hombres $(-49.0 \%)$ que entre las mujeres $(-45.1 \%)$. La mayor cantidad de muertes en eventos no intencionales se presentó en dos categorías: los accidentes de tráfico de vehículo de motor y las asfixias por inmersión, que en conjunto representaron $63.3 \%$ de las causas no intencionales.

\section{Homicidios}

Los homicidios muestran una tendencia ligeramente ascendente $(p=0.03$ ) de $9.5 \%$ a expensas de los grupos de 15 a 19 años de edad, y no en los de 10 a 14 . El ataque con armas de fuego se aprecia seguido por el ataque con instrumentos cortantes y punzantes, que en conjunto completan $72.9 \%$ de los homicidios.

\section{Suicidios}

Los suicidios son la categoría de traumatismos y envenenamientos con una tendencia ascendente $(p<0.01)$ que muestra el mayor cambio porcentual. Del trienio 1979-1981 al 1996-1997 la tasa de suicidios se incrementó en $104.0 \%$, observándose que fue durante la segunda mitad del periodo estudiado cuando los suicidios sufrieron su mayor crecimiento, pues el incremento de 1987-1989 a 1995-1997 fue de $74.6 \%$. Su tendencia ascendente se aprecia en uno $\mathrm{u}$ otro sexo, $\mathrm{y}$ es más acentuada en las mujeres de 10 a 14 años de edad, en las que el incremento de 1979-1981 a 19951997 llegó a 307.7\%. Este grupo es seguido por el de hombres de 10 a 14 años de edad en los que el incremento llegó a 142.6\%. En el grupo de 15 a 19 años de edad el incremento para mujeres y hombres fue de 60.4 y $93.1 \%$, respectivamente.

El ahorcamiento fue el medio que con más frecuencia se utilizó por los hombres, seguido por el uso de armas de fuego y el envenenamiento. Entre las mujeres el envenenamiento ocupó el primer lugar, seguido por el ahorcamiento y las armas de fuego. Esta composición en los medios para cometer suicidio se mantuvo más o menos constante durante el periodo. No obstante, se aprecian algunas tendencias que llaman la atención: el uso de armas de fuego entre las mujeres de 10 a 14 años de edad ha tenido un incremento de $600 \%$, aunque en la segunda mitad del periodo ha disminuido; entre las mujeres se observa un aumento en el uso del ahorcamiento (257.1\% en las de 15 a 19 años de edad, $400.0 \%$ en las de 15 a 19 ).

\section{Discusión}

Los resultados muestran una tendencia claramente descendente de los TyE que causan la muerte de adolescentes en México. No obstante, su tendencia aún sigue siendo un problema de salud pública que representa más de $50 \%$ de la mortalidad de adolescentes. La tendencia general está marcada por la gran cantidad y el comportamiento de los eventos mortales no intencionales que disminuyeron de manera importante 
durante el periodo estudiado, aunque los eventos intencionales (homicidios y suicidios) se incrementaron significativamente.

El comportamiento epidemiológico de la mortalidad por TyE que afecta a adolescentes mexicanos muestra lo que Plitponkarnpim y colaboradores ${ }^{5}$ describen dentro de los países con ingreso medio superior: las enfermedades infecto-contagiosas pierden su importancia como causa de muerte al tiempo que los TyE, aunque muestren una tendencia descendente, ocupan los primeros lugares en las causas de muerte. En la población estudiada los TyE representaron la primera causa de muerte durante todo el periodo, mientras que las enfermedades infecciosas y parasitarias pasaron del segundo al cuarto lugar (figura 2).

La tendencia general que muestra la mortalidad por TyE de adolescentes en México es difícil de explicar. Este fenómeno se aprecia en la mayoría de los países desarrollados ${ }^{6}$ y se atribuye, de manera general, a una mejora en las condiciones socioeconómicas de su población, y a la implantación de algunas acciones preventivas exitosas. Pero este no es el caso para nuestro país donde el producto interno bruto per cápita se ha mantenido más o menos constante alrededor de 13000 pesos de $1970^{7}$ durante los últimos 20 años. Dichas medidas podrían explicar parte de la disminución observada, aunque tales acciones preventivas no se han aplicado en todo el territorio.

Por otra parte, aunque es importante destacar una reducción de -41.5 en la mortalidad por TyE, también es importante señalar que muchos países, en donde este problema ha sido atendido eficazmente, han logrado reducciones mucho más importantes. Por ejemplo, ${ }^{6}$ en $\mathrm{R}^{\mathrm{e}} 1975$ y 1975 Alemania y México presentaron una tasa de mortalidad por TyE en menores de 14 años de edad de 28.4/100 000 y 29.3/100 000, respectivamente. De 1991 a 1995 Alemania la había reducido a 8.3/100 000 (un decremento de $-70 \%$ ), mientras que para México se reportó del 19.8/100 000 (para una disminución de sólo $-32.4 \%$ ).

Los trabajos que describen la mortalidad a partir de registros nacionales de defunción presentan algunas limitaciones que deben destacarse. En primer lugar, las muertes sólo representan la "punta del iceberg", y no reflejan el comportamiento de los TyE que, aunque pueden ser muy frecuentes, no producen muchas defunciones. Tal es el caso de algunas intoxicaciones, heridas con instrumentos cortantes o punzantes, y contusiones, por mencionar algunas. En segundo lugar, el llenado de los certificados de defunción es una gran fuente de error, porque a pesar de que generalmente son elaborados por personal calificado (médicos, en su gran mayoría) existen muchos errores, criterios equivocados, y deficiencias en el llenado de los mismos. Por último, estos trabajos no permiten identificar en detalle las causas de la mortalidad por TyE. No obstante, la información que nos proporcionan orienta las acciones de investigación, prevención y atención de los eventos más graves: aquellos que producen la mayor cantidad de muertes. Tomando en cuenta lo anterior haremos algunas consideraciones en relación con los eventos más frecuentes.

Los ATVM son los eventos que producen la mayor cantidad de muertes entre adolescentes, y gran cantidad de estos eventos se codifican en la categoría "no especificado". Llama la atención que esta categoría muestre una tendencia descendente, la cual podría indicar que durante el periodo estudiado la calidad del reporte en el certificado de defunción ha mejorado. Aunque siempre queda la duda de a qué categoría corresponden esos eventos "no especificados", estudios previos muestran que la gran mayoría son peatones. ${ }^{8-10}$ Esto difiere de lo observado en países más desarrollados donde son más numerosas las muertes de ocupantes, ${ }^{11,12}$ o la diferencia entre ambos peatones y ocupantes no es tan marcada ${ }^{13,14}$ como en México. Esto tiene grandes implicaciones en la prevención porque las intervenciones principalmente dirigidas a los ocupantes (cinturón de seguridad, bolsas de aire, modificaciones en la estructura de los vehículos) tienen poco impacto entre los peatones.

El arma de fuego ocupa el segundo lugar como causa de traumatismo. Su tasa de mortalidad está muy por debajo de lo reportado para Estados Unidos de América, ${ }^{11}$ donde la mortalidad en adolescentes de 15 a 19 años de edad llega a 27.8/100 000. Esta diferencia en la frecuencia de homicidios entre Estados Unidos de América y México también ha sido reportada por otros autores. ${ }^{15}$ La mortalidad observada en México se aproxima más a la reportada para Nueva Zelanda ${ }^{14} \mathrm{o}$ Australia. ${ }^{13}$ Sin embargo, en estos dos países se aprecian, con relación a México, diferencias importantes que deberían estudiarse: el número de suicidios en México es menor que el observado en Nueva Zelanda y Australia, con una razón de tasas de 0.24, mientras que el número de homicidios muestra ser mayor en México: 3.15 relativo a Australia, y 7.4 relativo a Nueva Zelanda.

Se reconoce que la disponibilidad de armas de fuego es un factor de riesgo de suicidio. ${ }^{16} \mathrm{Al}$ reducir la disponibilidad de armas entre adolescentes buscarían otros medios para suicidarse, como la sofocación y la intoxicación, aunque el número de muertes por estos mecanismos sería mucho menor que el esperado en condiciones en los que las armas de fuego están disponibles. Para el caso de los suicidios por ahorcamiento la mortalidad observada en México es supe- 
rior a la reportada para Estados Unidos de América, pero inferior a la de Nueva Zelanda y Australia. No obstante, para nuestro país es un problema prioritario de salud pública porque el número de suicidios está incrementándose, en parte a expensas de este mecanismo, que ocupa el primer lugar como forma de suicidio entre los hombres.

La prevención de las asfixias por inmersión representa un reto sin atender, particularmente cuanto éstas aparecen en el segundo o tercer lugar de las muertes traumáticas. ${ }^{17}$ En nuestro país ocupa la cuarta posición de la totalidad de los TyE mortales, y la segunda cuando sólo se toman en cuenta los eventos no intencionales. La tendencia observada muestra un descenso que no es posible explicar a partir de las bases de datos estudiados. No tenemos conocimiento de alguna intervención preventiva que se hubiera dirigido a adolescentes en México, pero de implantarse debería de tomar en cuenta dos hechos con relación a las asfixias por inmersión en adolescentes: estos eventos ocurren con mayor frecuencia en el campo (presas, lagos, ríos, canales); y cerca de 40\% de los ahogados de 15 a 19 años de edad presentan alguna concentración de alcohol en sangre. ${ }^{18}$

\section{Implicaciones para la prevención y la investigación}

No obstante las limitaciones que una investigación de este tipo tiene para el estudio de los TyE como causa de muerte, la revisión de la mortalidad de adolescentes por estas causas en México brinda información que puede ser utilizada para la atención de un problema de salud pública particularmente importante en este grupo de edad.

En primer lugar se destacan algunas causas de muerte que representan una gran cantidad de muertes por causas externas: los accidentes de tráfico de vehículo de motor y las asfixias por inmersión. En segundo lugar, destaca el gran incremento que los suicidios han tenido en los últimos 20 años y señala un tema que tendrá que ser atendido en un futuro inmediato. Estas tres causas de muerte traumática identifican tres áreas de investigación que deberán desarrollarse en México, principalmente porque la frecuencia de los eventos en los cuales se presentan los traumatismos mortales son diferentes a lo reportado en la literatura: quienes mueren con mayor frecuencia en eventos de tráfico son peatones y no ocupantes de vehículos, las asfixias por inmersión se presentan con mayor frecuencia en zonas no urbanas, y los medios que con mayor frecuencia se utilizan para cometer suicido son diferentes a las armas de fuego. Estas características de los eventos observados en México hacen que las intervenciones preventivas que han mostrado algún éxito en países desarrollados no logren los mismos resultados en México, al mismo tiempo obligan a incrementar la investigación de estos fenómenos en nuestro medio.

Por otra parte, la adolescencia es un periodo donde se establecen, de manera definitiva, una gran cantidad de actitudes y prácticas que influyen en el resto de la vida: aquellas actitudes y prácticas positivas que se introduzcan en la adolescencia impactarán positivamente el resto de nuestra existencia, de aquí la importancia de implantar acciones preventivas en dicho periodo.

\section{Referencias}

1. O rganización Panamericana de la Salud. Estadísticas de Salud de las Américas. W ashington, D.C ., O PS, 1998.

2. Instituto N acional de Estadística, Geografía e Informática. Información Estadística del Sector Salud y Seguridad Social.A guascalientes,Ags.:IN EG I, 1999, Cuaderno N úm. 15.

3. Rothman KJ, G reenland S. Moderm epidemiology.2da edición. Filadelfia: Lippinctt-Raven, 1998:31-35.

4. Kraus JF. Effectiveness of measures to prevent unintentional deaths of infants and children from suffocation and strangulation. Public Health Rep $1985 ; 100: 231-240$

5. Plitponkarnpin A, Andersson R, Jansson B, Svanström L. Unintentional injury mortality in children:A priority for middle income countries in the advanced stage of epidemiologycal transition. Inj Prev 1999; 5:98-103.

6. Chalmers D, Pless B. UN IC EF's child injury league tables:A bag of mixed messages. Inj Prev 2001; 7:81-82.

7. Instituto N acional de Estadística, G eografía e Informática. Estadisticas Históricas de México.Tomo I. 4th ed. A guascalientes,A gs.: IN EGI, 1999.

8. Celis A, Valencia N. Traumatismos y envenenamientos en Jalisco. Un estudio de mortalidad a partir de autopsias. Salud Publica Mex 1991; 33: 77-87.

9. Hijar M, Kraus J,Tovar V, C arrillo C . A nalysis of fatal pedestrian injuries in Mexico City, 1994-1997. Injury 2001; 32:279-284.

10. Híjar M. Mortalidad por lesiones accidentales e intencionales en el Distrito Federal. Salud Publica Mex 1990; 32:395-404.

11. Fingerhut L,Annest J, Baker S, Kochanek K, McLoughlin E. Injury mortality among children and teenagers in the United States, 1993. Inj Prev 1996; 2:93-94.

12. Scott I, Moller J, Bordeaux S. Injury mortality among children and teenagers in Australia, 1994. Inj Prev 1997; 3:46-47.

13. DiG uiseppi C, Roberts I. Injury mortality among children and teenagers in England and W ales, 1992. Inj Prev 1997; 3:47-49.

14. Langley J, Smeijers J. Injury mortality among children and teenagers in $\mathrm{N}$ ew Zealand compared with the United States of America. Inj Prev 1997; 3:195-199.

15. Híjar M, C hu L, Kraus J. Cross-national comparison injury mortality: Los Angeles County, California and Mexico City, Mexico. Int J Epidemiol 2000; 29:715-721.

16. Christoffel T, Gallagher S. Injury Prevention and Public Health. Practical knowledge, skills, and strategies. Gaithersburg (MD): Aspen Publication, 1999.

17. Pless B. The challenge of drowning prevention. Inj Prev 1997; 3: 237-238.

18. Celis A.A sfixia por inmersión en Jalisco: 1983-1989. Salud Publica Mex 1991; 33:585-589. 